\title{
Negative ethical behaviors in Saudi hospitals: How prevalent are they perceived to be? - Statement agreement study
}

\author{
R. Fayez, A. Nawwab' ${ }^{1}$, H. AL-Jahdali', S. Baharoon², S. Binsalih'², A. Al Sayyari ${ }^{2}$ \\ Department of Psychiatry, Armed Forces Hospital, ${ }^{1}$ Medical Student, College of Medicine, ${ }^{2}$ Medicine, King Saud Bin Abdulaziz University for \\ Health Sciences, Riyadh, Kingdom of Saudi Arabia
}

\begin{tabular}{|c|}
\hline Access this article online \\
\hline Website: www.avicennajmed.com \\
\hline DOI: 10.4103/2231-0770.118458 \\
\hline Quick Response Code: \\
\hline
\end{tabular}

\begin{abstract}
BACKGROUND: There is limited information about the prevalence of unethical behavior and how is perceived among health care providers. The aim of this study is to assess such behavior and how is perceived. MATERIALS AND METHODS: This is a cross-sectional study among three groups of professionals. Total participants were 370 and included medical staff, medical residents, and nurses in five medical specialties in four tertiary hospitals in Saudi Arabia (two Ministry of Health Hospitals and two military Hospitals). Participants were asked to rate their agreement with occurrence of 15 "negative" unethical behavior scenarios in their workplace. The scenarios covered areas of "respect for persons", "interprofessional relationships," and "empathy with patients". RESULTS: Majority of respondents agreed that "unethical" behavior occurred in their workplace, including confidentiality being compromised (36.3\%), informed consent not taken properly (60.2\%), and bad news not well-delivered (62.2\%). Other significant area agreement included doctors lacking empathy (47.8\%), patient autonomy not fully respected $(42.5 \%)$, discrimination $(41.2 \%)$, and being pressurized to write inaccurate reports $(31.2 \%)$. Respondents in medicine had the lowest rate of agreement and those in psychiatry had the highest (mean of $49.8 \%$ and $82.3 \%$, respectively). Respondents with length of employment of less than 6 years had significantly higher agreement that unethical behavior occurs compared to those with length of employment of more than 6 years. Males were more likely than females to agree that unethical behavior occurs. The biggest difference was seen in the behavior of "informed consent not properly taken" with a gender margin of $18.7 \%(P=0.001)$. CONCLUSION: There is high prevalence of behavior that is considered unethical as perceived by various health care workers at Saudi hospitals.
\end{abstract}

Key words: Bioethics, ethics in hospitals, Saudi Arabia

\section{INTRODUCTION}

Respect for persons, good interprofessional relationships, and empathy with patients are areas that are very important for optimal health care delivery. They also reduce the frequency of complaints by staff and patients and patients' relatives. ${ }^{[1]}$ There is also evidence from business ethics literature that the organization's commitment to ethics results in better job satisfaction and outcomes. ${ }^{[2]}$
Medical students report that they observe unethical behavior commonly by health care providers. In a survey in one USA hospital, $35 \%$ of $1^{\text {st }}$-year students reported having observed unethical behavior. This rose to $90 \%$ in $4^{\text {th }}$-year students. ${ }^{[3]}$ In another US survey, $98 \%$ of students had heard physicians refer to patients in a derogatory way and $61 \%$ had witnessed unethical incidents. ${ }^{[4]}$ This could lead to what has been termed "traumatic deidealization," ${ }^{[5]}$ whereby the students become cynical and lose their moral sensitivities. ${ }^{[6]}$ This is manifested by the finding that a third of medical students

Address for correspondence: Dr. Salih Bin Salih, Department of Medicine, King Saud Bin Abdulaziz University for Health Sciences,

King Abdulaziz Medical City, Riyadh, Kingdom of Saudi Arabia. E-mail: binsalihs@ngha.med.sa 
believed the derogatory comments made by physicians about the patients to be "sometimes" or "often" appropriate and the frequency of such beliefs among medical students increase with seniority. ${ }^{[7]}$

Health care organizations are now required by the Joint Commission on Accreditation of Healthcare Organizations to have defined standards of patient's rights and organizational ethics.

The increasing complexity of health care systems, treatment and diagnostic options demand concurrent development of ethical standards at patients' level, interpersonal level and organizational level. This requirement is no more acute than in the Saudi Arabian health system which developed from rudimentary medical services just a few decades ago to state of art medical services encompassing all types or complex services such as solid organ transplantation, conjoint twins' separation and treatment, and advanced intensive care units provisions of renal, ventilatory, cardiac, and neurological support in highly developed setting.

These developments coincided with two other important changes that have pertinent impacts on ethical behaviors and perceptions in the health care settings in the Saudi Arabian context. First, the Saudi society has become increasingly sophisticated, less tribal, more globalized, and more demanding and questioning. This prompted the health care organizations to be more patient-centered with increasing tendency to patient empowerment. Second, doctors training in Europe and North America as well as the largely expatriate physician and nursing workforce in Saudi Arabia bring with them beliefs regarding the pivotal importance of proper informed consent and the primacy of patient's autonomy which are new to the Saudi health care settings and which, in the past, tended to be patriarchal and paternalistic.

Very little has been written on ethical behaviors in hospitals in Saudi Arabia. ${ }^{[8]}$ This study aims to look into the ethical behavior among health care providers in Saudi hospitals.

\section{MATERIALS AND METHODS}

This is a cross-sectional self-administered survey-based study among three groups of professionals-nurses, medical residents, and medical staff. Respondents were chosen randomly among medical staff, medical residents, and nurses in five medical specialties in four tertiary hospitals in Saudi Arabia (two Ministry of Health Hospitals and two military Hospitals). The survey was distributed by two medical students during medical education setting and collected at the end of the education sessions. A total of
450 questionnaires were distributed and 370 questionnaires were collected giving the response rate of $82 \%$.

Respondents were asked to rate their agreement with occurrence of 15 "negative" unethical behavior scenarios in their workplace according to a five-scale scoring system: (1) Completely agree, (2) agree, (3) do not know, (4) disagree, and (5) completely disagree. The scenarios covered areas of "respect for persons" (five scenarios), "interprofessional relationships" (six scenarios), and "empathy with patients" (four scenarios). These survey questionnaires developed by investigators in English language and tested and validated among 30 participants.

Data were analyzed using descriptive statistics (frequency distribution and percentages).

The overall mean scores as well as scores for each scenario were calculated. The independent impact of age, gender, position, duration, and specialty on the three summative ethical domains was investigated using linear multivariate regression analysis. The responses were also analyzed in a dichotomous fashion grouping "very often" and "sometime" as "agree" (that the scenario occurs) and grouping "rarely" and "never" as "disagree" (that the scenario occurs). Chi-square test was used to compare proportions and percentages of responses. Analysis of variance was used to compare means between groups.

Ethical consent was obtained from the ethics committee and a covering letter was also distributed with the survey that described the purpose of the study, confidentiality of responses, and voluntary nature of the survey.

\section{RESULTS}

\section{Demographic data}

A total of 370 respondents were enrolled ( $82 \%$ of those approached). The mean age was $32.4( \pm 7.7)$ years and males constituted 208 (56.2\%) of the respondents. Half of the respondents were working in Ministry of Health Hospitals and the other half in Military Hospitals. Of the respondents, $(27.8 \%)$ were tenured medical staff, 166 (44.9\%) were residents, and 101 (27.3\%) were nurses. The respondents were working in the following specialties: 195 (52.7\%) in medicine, 68 (18.4\%) in surgery, 21 (5.7\%) in obstetrics and gynecology, 45 (12.2\%) in psychiatry and $41(11.1 \%)$ in pediatrics [Table 1].

\section{Rate of agreement on certain unethical behaviors scenarios}

Table 2 shows the overall rate of agreement on the occurrence of the 15 unethical behavior scenarios in descending 
order (i.e., from scenarios thought to occur more commonly to those thought less likely to occur). Majority of junior staff agreed regarding concerns of juniors staff not taken seriously (75.4\%), 58.6\% were concerned about physicians criticizing colleagues in front of juniors. Also $47.8 \%$ agreed regarding doctors lack empathy, $42.5 \%$ agreed that patient autonomy was not fully respected, $41.2 \%$ agreed that social discrimination occurs, and $31.2 \%$ agreed about being pressurized to write inaccurate reports. Respondents agreed that "unethical" scenarios occurred including confidentiality being compromised (36.3\%), informed consent not taken properly (60.2\%), and bad news not delivered well (62.2\%).

Respondents working in psychiatry were most likely and those working in medicine least likely to agree that negative

\begin{tabular}{|c|c|}
\hline Position & $\%$ \\
\hline Medical staff & 27.8 \\
\hline Residents & 44.9 \\
\hline Nurses & 27.3 \\
\hline \multicolumn{2}{|l|}{ Specialty } \\
\hline Medicine & 52.7 \\
\hline Surgery & 18.4 \\
\hline Pediatrics & II.I \\
\hline Obstetrics and gynecology & 5.7 \\
\hline Psychiatry & 12.2 \\
\hline Gender (male \%) & 56.3 \\
\hline Medical staff & 76 \\
\hline Residents & 43.3 \\
\hline Nurses & 14.5 \\
\hline \multicolumn{2}{|l|}{ Religion } \\
\hline Medical staff & 100 Moslem \\
\hline Residents & 100 Moslem \\
\hline Nurses & 27.I Moslem and 72.9 Christian \\
\hline Mean age (standard) years & $32.4 \pm 7.7$ years \\
\hline Under 30 years of age $n(\%)$ & $173(46.70)$ \\
\hline Over 30 years of age $n(\%)$ & $197(53.3)$ \\
\hline \multirow[t]{2}{*}{ Medical sector $n(\%)$} & Military 185 (50) \\
\hline & Ministry of Health I85 (50) \\
\hline
\end{tabular}

\begin{tabular}{lc}
\hline Table 2: Overall rate of agreement on certain unethical \\
behaviors scenarios in (\%) \\
\hline Juniors concerns on management not taken seriously \\
Poor bad news delivery & 75.40 \\
Informed consent not properly taken & 62.20 \\
Seniors do not consider teaching a part of duty & 60.20 \\
Physicians criticize colleagues in front of juniors & 59.30 \\
Doctors lack empathy & 58.60 \\
Psychosomatic patients resented & 47.80 \\
Patient autonomy not fully respected & 42.70 \\
Differential treatment (social discrimination occurs) & 42.50 \\
Confidentially not fully maintained & 41.20 \\
Pressurized to write inaccurate reports & 36.30 \\
DNR orders taken lightly & 31.20 \\
Treatment withdrawal occurs frequently & 31.10 \\
Female patients are discriminated against & 29.30 \\
Doctors expect gifts & 21.10 \\
*All percentages are rounded to one decimal, DNR: Do not resuscitate & 4.10 \\
\hline
\end{tabular}

behavior ethical behaviors occur. Those who were on the job $<6$ years are more likely than those $>6$ years to agree that unethical behaviors occur.

\section{Agreement by profession and gender}

Table 3 shows the impact of profession on the rate of agreement (only scenarios with significant differences are shown). It can be seen that without exceptions more medical residents are likely to agree that unethical behaviors occur than do medical staff or nurses.

When comparing response by male and females, we find significant difference in the agreement rate in 6 out of the 15 unethical behaviors. In all these, males were more likely than females to agree that unethical behavior occurs. The biggest difference was seen in "informed consent not properly taken", "social discrimination", and "poor bad news delivery" ( $P \leq 0.05)$ [Table 4].

\section{Rate of agreement by specialty}

When comparing the impact of specialty, we find that significant differences are seen in one-third of the scenarios. It is interesting to note that respondents in medicine had the lowest rate of agreement and those in psychiatry had the highest (mean of $49.8 \%$ and $82.3 \%$, respectively) others finding presented in [Table 5].

\begin{tabular}{|c|c|c|c|c|}
\hline & Medical & Residents & Nurses & $P$ \\
\hline Autonomy not fully respected & 41.7 & 53.9 & 23.7 & 0.000 \\
\hline Poor bad news delivery & 60.2 & 70.1 & 52.7 & 0.048 \\
\hline Confidentially not maintained & 34.4 & 48.8 & 16.1 & 0.000 \\
\hline $\begin{array}{l}\text { Physicians criticize colleagues in } \\
\text { front of juniors }\end{array}$ & 62.1 & 73.5 & 30.1 & 0.000 \\
\hline Doctors lack empathy & 39.8 & 62.2 & 33.3 & 0.000 \\
\hline $\begin{array}{l}\text { Female patients are } \\
\text { discriminated against }\end{array}$ & 14.6 & 29.1 & 14.0 & 0.000 \\
\hline $\begin{array}{l}\text { Seniors do not consider } \\
\text { teaching a part of duty }\end{array}$ & 56.9 & 84.3 & 20.4 & 0.000 \\
\hline $\begin{array}{l}\text { Juniors concerns on } \\
\text { management ignored }\end{array}$ & 76.7 & 89.8 & 52.7 & 0.000 \\
\hline Doctors expect gifts & 1.0 & 6.1 & 3.4 & $0.01^{\circ}$ \\
\hline Psychosomatic patients resented & 40.8 & 59.2 & 16.1 & 0.000 \\
\hline
\end{tabular}

\begin{tabular}{lccc}
\hline \multicolumn{4}{l}{ Table 4: Rate of agreement by gender in (\%). Only } \\
scenarios with significant differences are shown \\
\hline & Male & Female & P \\
\hline Informed consent not properly taken & 69.0 & 50.3 & 0.001 \\
Patient autonomy not fully respected & 67.2 & 50.3 & 0.007 \\
Differential treatment & 50.2 & 34.8 & 0.000 \\
(social discrimination occurs) & & & \\
Poor bad news delivery & 50.5 & 36.1 & 0.010 \\
Doctors lack empathy & 34.8 & 24.5 & 0.050 \\
Psychosomatic patients resented & 5.5 & 2.0 & 0.000 \\
\hline *All percentages are rounded to one decimal & \multicolumn{3}{l}{}
\end{tabular}


Fayez, et al.: Ethical behavior in Saudi hospitals

\begin{tabular}{|c|c|c|c|c|c|c|}
\hline & Medicine & Surgery & Pediatrics & Obs and Gyne & Psychiatry & $\mathbf{P}$ \\
\hline Informed consent not properly taken & 55.8 & 61.4 & 76.5 & 76.5 & 76.0 & 0.02 \\
\hline Physicians criticize colleagues in front of juniors & 49.7 & 64.9 & 64.7 & 64.7 & 82.0 & 0.001 \\
\hline Patient autonomy not fully respected & 34.1 & 43.9 & 44.1 & 70.6 & 63.0 & 0.03 \\
\hline Patients with psychosomatic symptoms are derided & 40.6 & 43.9 & 20.6 & 64.7 & 84.2 & 0.000 \\
\hline Seniors do not consider teaching a part of duty & 51.5 & 73 & 67.6 & 70.6 & 87 & 0.004 \\
\hline Juniors concerns on management ignored & 67 & 89 & 100 & 100 & 100 & 0.000 \\
\hline
\end{tabular}

*All percentages are rounded to one decimal, Obs and Gyne: Obstetrics and Gynecology

\section{Rate of agreement by length of employment}

Respondents who spent 6 or less years on the job were more likely than those who spent more than 6 years to agree that certain unethical behaviors occur, including the areas of empathy, autonomy, and confidentiality and there is a tendency toward disagreeing with the statements that unethical behaviors occur as the duration on the job increase [Table 6].

\section{Summation of the scenarios into three domains}

We further analyzed the data after summation of the 15 scenarios into three domains, "Respect for Persons", "Empathy," and "Professionalism" as shown in Table 7. Overall, $42.9 \%$ of all respondents indicated their agreement with the existence of unethical behavior in these domains. This can be broken down into $38.5 \%$ for "respect for persons" domain, $45.7 \%$ for "empathy" domain, and $45.9 \%$ for "professionalism" domain [Table 8]. Using linear multivariate regression analysis, we found no independent impact of age, gender, nationality, specialty, or length of experience on any of the three domains.

\section{DISCUSSION}

Ethical behavior by health care staff is influenced by a number of factors. These include observed peer ethical behavior, ${ }^{[9]}$ mentor influence, ${ }^{[10]}$ gender, ${ }^{[11]}$ and previous training in ethics as well as the ethical environment and culture in the organization. ${ }^{[1]}$

Doctors and nurses differ in the emphasis and weight they give to different ethical principles. In one recent study in USA, nurses were found to be significantly more ethical than other employees. ${ }^{[9]}$ Specifically, nurses tend to give greater weight to virtues and patient autonomy, whereas doctors tend to invoke beneficence. ${ }^{[12]}$ In our study, however, more doctors than nurses agreed that patients' autonomy is not respected. The nurses in this study differed from the physicians in three aspects: The majority of them were female, Christian and from developing countries. Any of the above can explain the finding seen in this study regarding the view of physicians and nurses. This is different from differ from published studies in North America. ${ }^{[1]}$

\begin{tabular}{|c|c|c|c|}
\hline $\begin{array}{l}\text { Table 6: Rate of agreement by le } \\
\text { in (\%). Only scenarios with signif }\end{array}$ & $\begin{array}{l}\text { gth of em } \\
\text { ant differc }\end{array}$ & $\begin{array}{l}\text { ployment } \\
\text { nces are }\end{array}$ & \\
\hline & $<6$ years & $>6$ years & $\mathbf{P}$ \\
\hline $\begin{array}{l}\text { Senior staff feel teaching is not part of } \\
\text { the job }\end{array}$ & 77 & 49 & 0.0001 \\
\hline Female patients are discriminated against & 27.0 & 15.5 & 0.02 \\
\hline $\begin{array}{l}\text { Senior physicians criticize each other in } \\
\text { front of junior staff }\end{array}$ & 69.1 & 53.8 & 0.012 \\
\hline Doctors are not empathic & 59.0 & 41.7 & 0.001 \\
\hline Autonomy of patients is not respected & 53.4 & 37.3 & 0.004 \\
\hline $\begin{array}{l}\text { Patients with psychosomatic symptoms } \\
\text { are derided }\end{array}$ & 52.8 & 37.2 & 0.007 \\
\hline Confidentially not fully maintained & 46.6 & 29.7 & 0.006 \\
\hline
\end{tabular}

\begin{tabular}{lll}
\hline \multicolumn{2}{l}{ Table 7: Summation of the I5 scenarios into three } \\
domains
\end{tabular}

Table 8: Agreement and disagreement in (\%) (5 I 53 responses in 370 patients)

\begin{tabular}{lccc}
\hline Domain & $\begin{array}{c}\text { Agree } \\
\text { (\%) }\end{array}$ & $\begin{array}{c}\text { Disagree } \\
\text { (\%) }\end{array}$ & $\begin{array}{c}\text { Do not know } \\
\text { (\%) }\end{array}$ \\
\hline Suboptimal "Respect for persons" & 38.5 & 60.2 & 1.4 \\
Suboptimal "Empathy" & 45.7 & 51.8 & 2.5 \\
Could do better "Professionalism" & 45.9 & 52.1 & 2 \\
All domains & 42.9 & 55.4 & 1.7 \\
\hline *All percentages are rounded to one decimal & &
\end{tabular}

A study by Munro and Powis suggests two important personality dimensions as influencing ethical behavior. These are narcissism (related to disagreeableness, aggressiveness, aloofness from others, sensitivity to rewards, and anxiety) and empathy (related positively to emotional intelligence, extroversion, open-mindedness, compliance with others, and not being aloof). ${ }^{[13]}$ Previous studies have shown that nurses, females in general, and primary care physicians 
have more empathetic personality, whereas surgeons tend to have aggressive and aloof personalities which could affect their ethical perceptions and behavior. ${ }^{[14]}$ In the present study, we found that, among the various specialties, the psychiatrists had the highest agreement with the statements about unethical behavior occurring in the workplace, with the least agreement noted among respondents from medicine specialty. It is unlikely that this reflects more unethical behavior within psychiatry practice. The reason for this is not clear, but we speculate that psychiatrists are perhaps more ethics-sensitive than other specialists due to the nature of their profession. The duration on the job is increasingly associated with reduced reporting of the occurrence of unethical events. When comparing those who were on the job for more than 6 years to those less than 6 years, we found that significantly higher numbers of the latter group agreeing that unethical behavior occurs. This might be related to more acceptance of unethical behavior as time passes on the job rather than younger staff perceiving subethical behavior when it does not exist. Previous studies involving medical students in North America have revealed reduced moral sensitivity occurring with the passage of time during the medical training and a hidden curriculum was questioned. ${ }^{[15]}$ Surprisingly, male respondents were found to be more likely to agree that unethical behavior occurs than female respondents. The biggest difference was seen in "informed consent not properly taken" with a margin of $18.7 \%(P=0.001)$. This is contrary to a previous study we did on professional boundary ethics in which we found that females tend to be more "ethically" stringent than males. ${ }^{[16]}$ Previous studies have also found higher ethical standards and moral sensitivity among women. ${ }^{[11]}$

Many previous literature reports on business ethics found differences in ethical beliefs between women and men. Betz et al., ${ }^{[17]}$ for example, found that men are likely to engage in unethical practices. In contrast, some other studies found no gender differences in ethical attitudes. ${ }^{[18]}$ Satish et al. ${ }^{[9]}$ also found that the race of the respondent did not impact ethical behavior.

This paper brings forth a number of findings that we think are of relevance and importance. First, senior staffs do not give enough importance or spend sufficient time discussing or reflecting on ethical issues related to patients. This has been attributed in previous reports to senior staff not feeling that there is a need to teach ethics formally. ${ }^{[19]}$ They often feel that ethics is intuitional and does not require specific formal and organized instruction. This belief is contrary to the repeated findings which show that medical students' moral sensitivity falls as the medical course progresses.
Studies have shown that moral sensitivity of medical student's falls as the medical training progresses, this attributed probably to medical students being affected or exposed to unethical behavior by their peers and seniors. ${ }^{[20,21]}$

The second important finding of this study is that residents were two to three times more likely than medical staff to agree that unethical behaviors occur. This follows from what was mentioned above, that is, higher degrees of moral sensitivities are observed initially in medical training, and which then drop with time.

Third, we believe the most interesting finding is that duration on the job is increasingly associated with less perception of negative ethical behavior. This is in keeping with previous findings ${ }^{[22-24]}$ and might be related to progressive falling of moral sensitivities from observing suboptimal ethical behavior of peers and seniors. ${ }^{[20]}$

\section{CONCLUSIONS}

Unethical behavior occurs in the workplace at Saudi hospitals and related to many factors. To reduce unethical behavior in hospitals, it is essential that organizations develop a strong ethical culture. Health care organizations should conduct seminars and workshops for health care professionals to discus different aspects of ethical issues including the issues that we investigated in this study. Further studies on factors that affect unethical behavior in health care organizations are needed to address the prevalence of unethical behavior in Saudi hospitals.

\section{REFERENCES}

1. Deshpande SP, George E, Joseph J. Ethical climates and managerial success in Russian organizations. J Bus Ethics 2000;23:211-7.

2. Hian Chye Koh EfHYB. Organisational ethics and employee satisfaction and commitment. Manage Decision 2004;42:677-93.

3. Satterwhite WM $3^{\text {rd }}$, Satterwhite RC, Enarson CE. Medical students' perceptions of unethical conduct at one medical school. Acad Med 1998;73:529-31.

4. Feudtner C, Christakis DA, Christakis NA. Do clinical clerks suffer ethical erosion? Students' perceptions of their ethical environment and personal development. Acad Med 1994;69:670-9.

5. Kay J. Traumatic deidealization and the future of medicine. JAMA 1990;26;263:572-3.

6. Wolf TM, Balson PM, Faucett JM, Randall HM. A retrospective study of attitude change during medical education. Med Educ 1989;23:19-23.

7. Satterwhite RC, Satterwhite WM $3^{\text {rd }}$, Enarson C. An ethical paradox: The effect of unethical conduct on medical students' values. J Med Ethics 2000;26:462-5.

8. Saeed KS. How physician executives and clinicians perceive ethical issues in Saudi Arabian hospitals. J Med Ethics 1999;25:51-6.

9. Satish P, Deshpande JJ. Factors impacting ethical behavior in hospitals. J Bus Ethics 2006;69:207-16.

10. Weaver GR, Trevino LK, Agle B. 'Somebody I look up to:' Ethical role models in organizations. Organ Dyn 2005;34:313-30. 
11. Perryer C, Jordan C. The influence of gender, age, culture and other factors on ethical beliefs: A comparative study in Australia and Singapore. Public Adm Manage: An Interactive J 2002;7:367-82.

12. Robertson DW. Ethical theory, ethnography, and differences between doctors and nurses in approaches to patient care. J Med Ethics 1996;22:292-9.

13. Don Munro MB, Powis D. Personality factors in professional ethical behaviour: Studies of empathy and narcissism. Aust J Psychol 2005;57:49-60.

14. Hejaili F, Juhani A, Flaiw A, Ghamdi G, Jondeby M, Eid A, et al. Is there a bias against women in kidney transplantation practices in Saudi Arabia? Exp Clin Transplant 2006;4:571-3.

15. Kittmer T, Hoogenes J, Pemberton J, Cameron BH. Exploring the hidden curriculum: A qualitative analysis of clerks' reflections on professionalism in surgical clerkship. Am J Surg 2013;205:426-33.

16. Tamim FH, Jamal A, Al Shamsi H, Al Sayyari A. Professional boundary ethics attitudes and awareness among nurses and physicians in a university hospital in the Kingdom of Saudi Arabia. Ethics Behav 2010;20:21-32.

17. Betz M, O'Connell L, Shepard J. Gender differences in proclivity for unethical behavior. J Bus Ethics 1989;8:321-4.

18. Costa AS. Are women more ethical than men? J Bus Ethics 1994;13:859-71.

19. Lempp H, Seale C. The hidden curriculum in undergraduate medical education: Qualitative study of medical students' perceptions of teaching. BMJ 2004;329:770-3.

20. Cordingley L, Hyde C, Peters S, Vernon B, Bundy C. Undergraduate medical students' exposure to clinical ethics: A challenge to the development of professional behaviours? Med Educ 2007;41:1202-9.

21. Caldicott CV, Faber-Langendoen K. Deception, discrimination, and fear of reprisal: Lessons in ethics from third-year medical students. Acad Med 2005;80:866-73.

22. Branch WT Jr. Supporting the moral development of medical students. J Gen Intern Med 2000;15:503-8.

23. Patenaude J, Niyonsenga T, Fafard D. Changes in students' moral development during medical school: A cohort study. CMAJ 2003;168:840-4.

24. Mattick K, Bligh J. Teaching and assessing medical ethics: Where are we now? J Med Ethics 2006;32:181-5.

Cite this article as: Fayez R, Nawwab A, Al-Jahdali H, Baharoon S, Binsalih S, AI Sayyari A. Negative ethical behaviors in Saudi hospitals: How prevalent are they perceived to be? - Statement agreement study. Avicenna J Med 2013;3:57-62

Source of Support: Nil, Conflict of Interest: None declared.

\section{“QUICK RESPONSE CODE” LINK FOR FULL TEXT ARTICLES}

The journal issue has a unique new feature for reaching to the journal's website without typing a single letter. Each article on its first page has a "Quick Response Code". Using any mobile or other hand-held device with camera and GPRS/other internet source, one can reach to the full text of that particular article on the journal's website. Start a QR-code reading software (see list of free applications from http://tinyurl.com/yzlh2tc) and point the camera to the QR-code printed in the journal. It will automatically take you to the HTML full text of that article. One can also use a desktop or laptop with web camera for similar functionality. See http://tinyurl.com/2bw7fn3 or http://tinyurl.com/3ysr3me for the free applications. 